\title{
INFLUÊNCIA DA PRÁTICA ESPORTIVA E DA IDADE NO DESENVOLVIMENTO DA COORDENAÇÃO MOTORA E DA APTIDÃO FÍSICA DE ESCOLARES DE FORTALEZA/CE
}

\author{
MARCELA DE CASTRO FERRACIOLI'; LARA DE SOUZA NUNES ${ }^{1}$
}

${ }^{1}$ Grupo de Pesquisa em Biodinâmica do Movimento Humano, Instituto de Educação Física e Esportes, Universidade Federal do Ceará - Fortaleza/CE - Brasil

Autor para correspondência:Marcela de Castro Ferracioli Endereço: Av. Mister Hull, Parque Esportivo - Bloco 320, Campus do Pici. CEP - 60455-760 - Fortaleza/CE Brasil

E-mail: marcelaferracioli@ufc.br Telefone: 55(85) 33669533

\section{AT A GLANCE}

Analisamos capacidades motoras e físicas de crianças praticantes e não praticantes de atividades esportivas extracurriculares, de diferentes idades. As crianças mais velhas tendem a ter melhor desempenho do que as mais novas. As crianças praticantes de esportes tendem a ter melhor desempenho do que as não praticantes apenas em componentes motores e físicos vivenciados nas modalidades esportivas.

\section{ABBREVIATIONS}

CE Ceará

F feminino

M masculino

MABC Movement Assessment Battery for Children

PROESP-BR Projeto Esporte Brasil
BACKGROUND: A literatura aponta que a prática esportiva pode potencializar as capacidades motoras e físicas das crianças em desenvolvimento e que, apesar disso, crianças que não praticam tais atividades podem apresentar desenvolvimento motor típico.

OBJETIVO: Analisar a influência da prática esportiva, enquanto estímulos ambientais, e da idade, enquanto processos desenvolvimentais, na coordenação motora e aptidão física de crianças.

MÉTODO: Participaram do estudo 80 crianças, sendo 20 de cada idade: 7, 8, 9 e 10 anos. Dez crianças de cada idade praticavam atividades esportivas extracurriculares e 10 praticavam apenas aulas de Educação Física Escolar. As crianças foram submetidas à avaliação da coordenação motora e da aptidão física. $O$ desempenho das crianças em cada componente motor e físico avaliado foi comparado entre as idades e entre grupos praticantes e não praticantes de atividades esportivas.

RESULTADOS: $O$ fator idade influenciou o desempenho das crianças na maioria dos componentes avaliados, enquanto a prática esportiva influenciou o desempenho nos componentes Equilíbrio e Força de Membros Inferiores apenas.

CONCLUSÃO: A prática esportiva influencia apenas componentes físicos e motores que são vivenciados na modalidade praticada. Apesar de os processos desenvolvimentais influenciarem o desenvolvimento motor, as crianças não estão apresentando níveis saudáveis de componentes da aptidão física.

\section{INTRODUÇÃO}

Por muitas décadas, estudiosos da área de Desenvolvimento Motor têm buscado identificar as mudanças motoras que ocorrem ao longo do tempo. Atualmente, o foco dessa área tem sido, principalmente, identificar os fatores que dão origem a essas mudanças durante a vida ${ }^{1}$. 0 desenvolvimento motor é dinâmico, tendo em vista que o mesmo muda em função das alterações maturacionais do sistema biológico e da interação do indivíduo com seu ambiente e com as características específicas de cada tarefa motora realizada ${ }^{2,3}$.

De interesse para o presente estudo são as mudanças decorrentes da prática de esportes enquanto restrições ${ }^{2}$ específicas da tarefa e do ambiente no desenvolvimento motor de crianças. Há consenso na área da Educação Física sobre os benefícios da prática esportiva para o desenvolvimento motor ${ }^{1,4,5}$, da saúde ${ }^{6,7}$ e psicosocial ${ }^{8}$. Em específico para o desenvolvimento da coordenação motora e da aptidão física, estudos mostraram que

\begin{tabular}{l|l|l|l|}
\hline Ferracioli \& Nunes & 2018 & VOL. 12 & N.1
\end{tabular}


Brazilian Journal of Motor Behavior

crianças praticantes de modalidades esportivas têm desempenho elevado em tarefas que requerem elementos motores e físicos encontrados na modalidade esportiva praticada. Por exemplo, Nazário e Vieira ${ }^{3}$ mostraram que crianças praticantes de handebol apresentam desempenho superior ao de crianças praticantes de futsal e ginástica artística nas tarefas que envolvem saltar com um pé e arremessar, enquanto crianças praticantes de futsal apresentam desempenho superior ao de crianças praticantes de handebol e de ginástica artística em tarefas que envolvem chutar e corrida lateral.

Outros estudos mostraram que crianças praticantes de atividade de futsal, ballet ${ }^{5} \mathrm{e}$ judô ${ }^{9}$ apresentaram desempenho superior na avaliação da coordenação motora quando comparadas com crianças que não praticaram essas atividades. No entanto, nesses estudos, as crianças não praticantes das atividades esportivas sistematizadas não apresentavam déficits ou dificuldades motoras, ou seja, mesmo não praticando as atividades sistematizadas, o desenvolvimento motor da criança parece ser típico.

Benefícios da prática esportiva sobre o desenvolvimento da aptidão física também são observados em testes das capacidades físicas requeridas na modalidade praticada. Machado Filho, Pelleginotti e Gonelli10 mostraram que a prática de handebol, futsal e atividades físicas gerais melhorou as capacidades físicas relacionadas à saúde de crianças. Outros estudos mostraram que crianças melhoraram as capacidades força de membros inferiores após prática sistematizada de futsal ${ }^{11}$ e que crianças que praticam essa modalidade têm melhor desempenho em testes de agilidade e velocidade do que as crianças que não praticam ${ }^{12}$. Apesar disso, crianças que não praticaram as atividades esportivas sistematizadas apresentavam capacidades da aptidão física satisfatórias, considerando a idade.

De maneira geral, foi possível perceber que a prática esportiva pode potencializar as capacidades motoras e físicas das crianças em desenvolvimento. No entanto, também se observou que as crianças que não praticaram atividades esportivas sistematizadas, nos estudos citados acima, apresentaram desenvolvimento típico. É nesse contexto que surge a questão referente ao quanto a prática de atividades sistematizadas (em específico, as esportivas) influencia o desenvolvimento motor. Seria o desenvolvimento da aptidão física e da coordenação motora dependente de processos desenvolvimentais, como àqueles associados ao aumento da idade, ou de processos decorrentes da prática de atividades específicas? Ainda, as capacidades físicas e motoras são desenvolvidas da mesma forma ou esses processos têm efeitos apenas sobre algumas? Assim, o presente estudo buscou investigar a influência da idade, enquanto processos desenvolvimentais inerentes ao organismo, e da prática esportiva extracurricular, enquanto características inerentes ao ambiente, nos componentes da aptidão física e da coordenação motora de escolares da cidade de Fortaleza/CE.

\section{MÉTODOS}

\section{Participantes}

Oitenta crianças de escolas públicas e particulares de Fortaleza/CE foram selecionadas por conveniência, sendo 20 crianças de cada uma das idades 7, 8, 9 e 10 anos, e separadas em dois grupos pareados por gênero e idade: Grupo Praticantes, com

\begin{tabular}{l|l|l|l|l}
\hline Ferracioli \& Nunes & 2018 & VOL. 12 & N.1 &
\end{tabular}


Brazilian Journal of Motor Behavior

40 crianças (20 meninos e 20 meninas) praticantes de modalidades esportivas extracurriculares há, no mínimo, quatro meses (Tabela 1); e Grupo Não-Praticantes, com 40 crianças praticantes apenas das aulas de Educação Física Escolar. As crianças e seus responsáveis consentiram a participação no estudo através da assinatura dos Termos de Assentimento do Menor e de Consentimento Livre e Esclarecido. Ambos os termos e todos os procedimentos do estudo foram aprovados pelo comitê de ética e pesquisa do Hospital Universitário Walter Cantídio da Universidade Federal do Ceará.

Tabela 1. Modalidades esportivas praticadas pelos participantes do Grupo Praticantes.

\begin{tabular}{ccccc}
\hline \hline & & \multicolumn{3}{c}{ Idade } \\
\hline Gênero & $\mathbf{7}$ anos & $\mathbf{8}$ anos & 9 anos & 10 anos \\
\hline F & Karatê & Natação & Ballet & Triatlon \\
F & Ballet & Jiu Jitsu & Natação & Natação \\
F & Natação & Natação & Natação & Natação \\
F & Natação & Jiu Jitsu & Natação & Karatê/Muay Thay \\
F & Natação & Natação & Natação & Ballet/Karatê \\
M & Futebol & Futebol & Futebol/Futsal & Natação \\
M & Natação & Natação/Futebol & Natação & Capoeira/Futebol \\
M & Natação & Basquetebol & Futebol & Natação \\
M & Karatê & Natação & Voleibol & Karatê \\
M & Karatê & Futebol & Futebol & Futebol/Jiu Jitsu \\
\hline \hline F= feminino; M= masculino & & &
\end{tabular}

\section{Aparatos e Tarefa}

Foram utilizados os materiais originais da Movement Assessment Battery for Children (MABC-2) ${ }^{13}$ para realização da avaliação da coordenação motora. Para a avaliação da aptidão física, foram utilizados: colchonete e banco de Wells para o teste Sentar e Alcançar; trena presa ao solo, colchonete e medicine ball $(2 \mathrm{~kg})$ para 0 teste Arremesso da Medicine ball; trena presa ao solo para o teste Salto Horizontal; colchonete e cronômetro para o teste Abdominal em 1 minuto; e cronômetro para 0 teste Corrida de 9 minutos. Estes materiais são indicados no manual do Projeto Esporte Brasil (PROESP$\mathrm{BR}^{14,15}$ ).

\section{Instrumentos}

As avaliações foram realizadas na quadra da escola em que a criança estava matriculada, pelas pesquisadoras responsáveis pelo estudo. Primeiramente, as crianças foram avaliadas individualmente através da MABC-2, composta por oito testes divididos em três componentes motores 13: Destreza Manual, Arremessar e Receber e Equilibrio Estático e Dinâmico. Foram utilizados os testes: Colocando pinos, Passando o cordão e 
Desenhando o caminho para avaliar Destreza Manual; Recebendo com as duas mãos e Arremessando o saco de feijão sobre 0 tapete para avaliar Arremessar e Receber; e Equilibrando sobre a prancha, Caminhando calcanhar-ponta para frente e Saltitando sobre tapetes para avaliar Equilíbrio. Após análise do escore obtido nessa avaliação, as crianças foram avaliadas através dos testes de aptidão física ${ }^{14}$. Foram utilizados os testes: Sentar e Alcançar para avaliar Flexibilidade; Arremesso de medicine ball para avaliar Força de Membros Superiores; Abdominal em 1 minuto para avaliar Resistência Muscular Localizada; Salto Horizontal para avaliar Força de Membros Inferiores; e Corrida de 9 minutos para avaliar Resistência Aeróbia.

\section{Análise Estatística}

Os dados da avaliação da coordenação motora foram registrados em planilha eletrônica configurada para conversão dos dados de desempenho em escores. Assim, foi obtida a pontuação e o percentil de classificação da criança, segundo a MABC-2 ${ }^{13}$. A criança era identificada com Desenvolvimento Típico se sua pontuação fosse igual ou superior ao $16^{\circ}$ percentil; com Risco de dificuldade motora se sua pontuação fosse entre $05^{\circ}$ e $15^{\circ}$ percentil; ou com Dificuldade Motora se a pontuação fosse igual ou inferior ao $5^{\circ}$ percentil. Para análises estatísticas, foram utilizados os percentis alcançados pelas crianças em cada componente motor e na avaliação total pela MABC-2. Além disso, foi descrita a frequência relativa de crianças com percentil abaixo do $16^{\circ}$ na MABC-2. Os dados da avaliação da aptidão física foram registrados em planilha eletrônica e utilizados para análise estatística. Além disso, foram utilizados os pontos de corte para valores normais de cada componente da aptidão física para identificar a frequência relativa de crianças com desempenho abaixo do Saudável14.

Foi feita Análise de Variância 2 (Grupo: Praticantes e Não-Praticantes) X 4 (Idade: $7,8,9$ e 10), com medidas repetidas no último fator, para cada componente motor e físico avaliado. $O$ teste post hoc de Tukey foi utilizado para identificar diferenças significativas. Foi considerado nível de significância $p<0,05$. Foi utilizado o software Statistica 7.0.

\section{RESULTADOS}

Os resultados dos componentes da coordenação motora são apresentados na Tabela 2 e dos componentes da aptidão física na Tabela 3.

Tabela 2. Média e desvio padrão dos percentis alcançados pelas crianças dos grupos Praticantes e Não-Praticantes de $7,8,9$ e 10 anos de idade.

\begin{tabular}{|c|c|c|c|c|c|c|c|}
\hline & Grupo & 7 anos & 8 anos & 9 anos & 10 anos & Total & \\
\hline \multirow{3}{*}{$\begin{array}{c}\text { Destreza } \\
\text { Manual }\end{array}$} & Praticantes & $25,1(17,4)$ & $31,5(27,5)$ & $41,1(28,5)$ & $52,7(20,5)$ & $37,6(25,3)$ & \multirow{3}{*}{$p=0,05$} \\
\hline & Não-Pratic. & $57,6(30,9)$ & $25,7(14,4)$ & $41,8(30,4)$ & $65,5(27,5)$ & $47,6(29,9)$ & \\
\hline & Total & $41,3(29,6)$ & $28,6(21,5)$ & $41,4(28,7)$ & $59,1(24,5)$ & & \\
\hline \multicolumn{8}{|c|}{$p<0,01$} \\
\hline \multirow{2}{*}{$\begin{array}{l}\text { Arremessar } \\
\text { e Receber }\end{array}$} & Praticantes & $23,7(20,1)$ & $21,5(15,6)$ & $39,3(22,1)$ & $40,5(30,0)$ & $31,2(23,4)$ & \multirow{2}{*}{$p=0,3$} \\
\hline & Não-Pratic. & $38,6(19,4)$ & $34,1(21,3)$ & $32,6(22,7)$ & $42,3(24,3)$ & $36,9(21,5)$ & \\
\hline
\end{tabular}




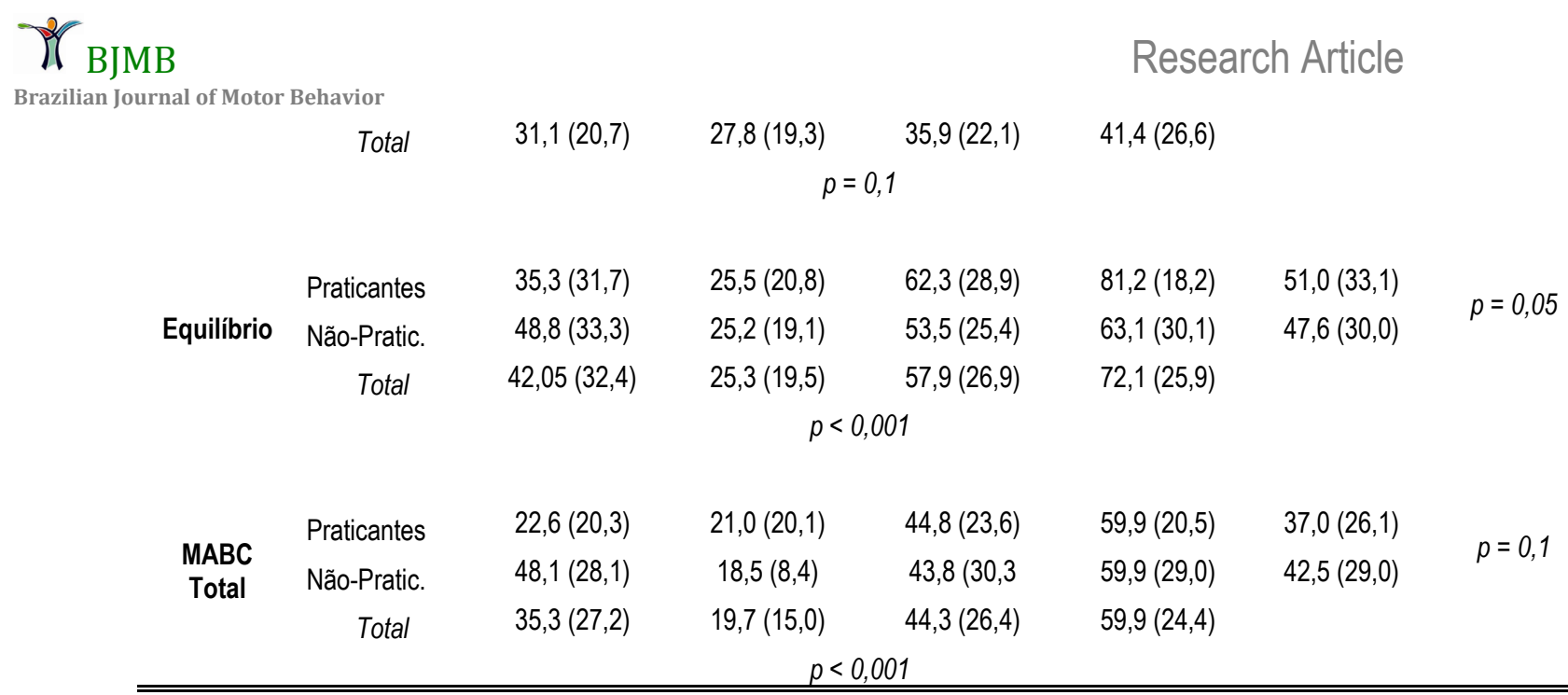

Destreza Manual. 0 fator Grupo apresentou diferença marginalmente significativa, $F(1,18)=4,1755, p=0,05$, e 0 fator Idade alcançou nível de significância, $F(3,54)=4,5201$, $p<0,01$. O Grupo Não-Praticantes apresentou maior percentil do componente Destreza Manual do que 0 Grupo Praticantes. Além disso, as crianças de 10 anos apresentaram maior percentil nesse componente $\left(59,1^{\circ}\right)$ do que as de $7\left(41,3^{\circ}\right)$ e 9 anos $\left(41,4^{\circ}\right)$, e todas apresentaram maior percentil do que as de 8 anos $\left(28,6^{\circ}\right)$.

Arremessar e Receber. Os fatores Grupo, Idade e a interação entre esses fatores não alcançaram nível de significância.

Equilibrio. Os fatores Grupo e Idade alcançaram nível de significância, $F(1,18)=0,35565, \quad p=0,05$ e $F(3,54)=11,280, \quad p<0,0001$, respectivamente. O Grupo Praticantes apresentou maior percentil do componente Equilíbrio do que o Grupo NãoPraticantes. Além disso, as crianças de 10 anos $\left(72,1^{\circ}\right)$ apresentaram maior percentil nesse componente do que as de $7\left(42,0^{\circ}\right)$ e 8 anos $\left(25,3^{\circ}\right)$, e essas últimas apresentaram menor percentil em relação às crianças de 9 anos $\left(57,9^{\circ}\right)$.

MABC-2 total. Apenas o fator Idade alcançou nível de significância, $F(3,54)=8,7568$, $p<0,0001$. As crianças de 10 anos $\left(59,9^{\circ}\right)$ apresentaram maior percentil total na MABC-2 do que as de $7\left(25,3^{\circ}\right)$ e 8 anos $\left(19,7^{\circ}\right)$, e essas últimas apresentaram menor percentil em comparação às crianças de 9 anos $\left(44,3^{\circ}\right)$.

Tabela 3. Média e desvio padrão dos resultados alcançados pelas crianças dos grupos Praticantes e Não-Praticantes de 7, 8, 9 e 10 anos de idade nos testes da avaliação da aptidão física.

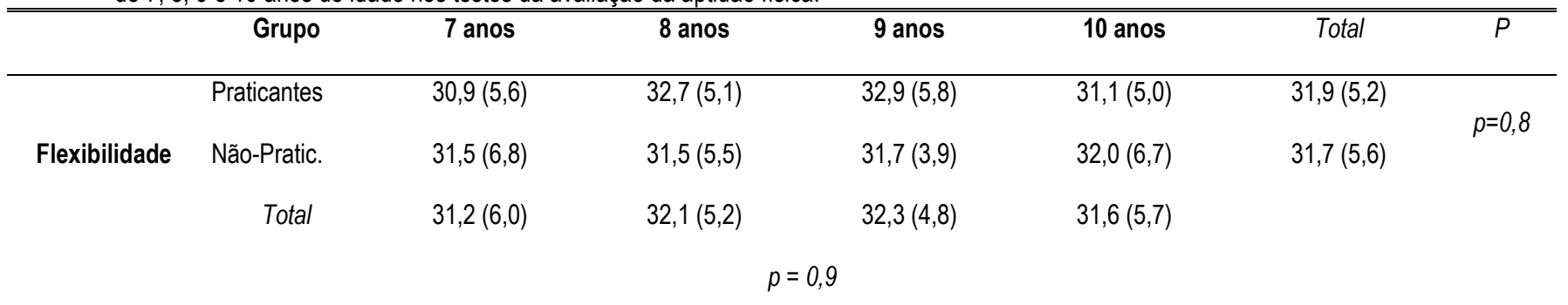

\begin{tabular}{l|l|l|l|l}
\hline Ferracioli \& Nunes & 2018 & VOL. 12 & N.1 & \\
& & & &
\end{tabular}




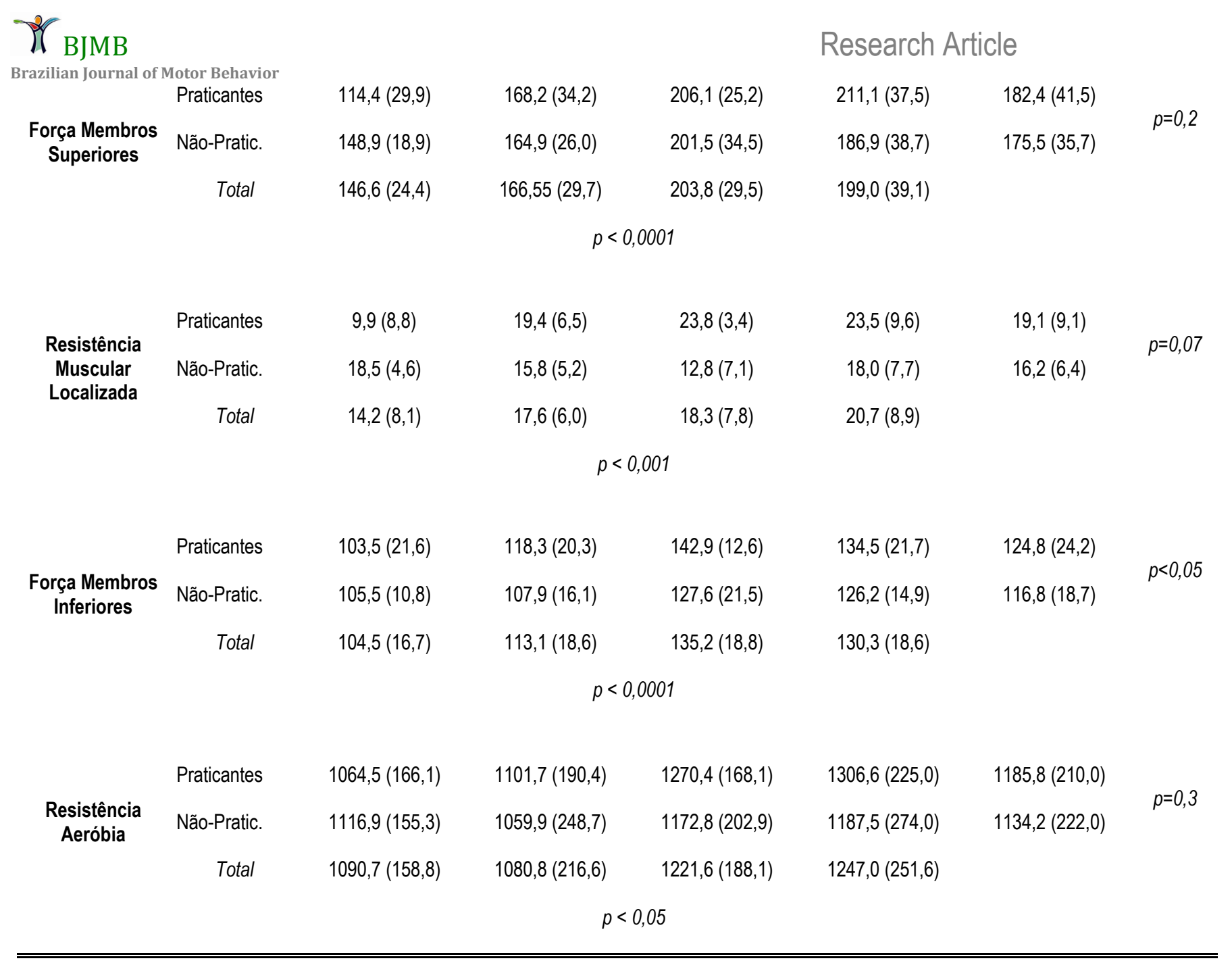

Flexibilidade. Os fatores Grupo, Idade e a interação entre esses fatores não alcançaram nível de significância.

Força de Membros Superiores. Apenas o fator Idade alcançou nível de significância, $F(3,54)=13,647, p<0,00001$. As crianças de $9(203,8 \mathrm{~cm})$ e 10 anos $(199,0 \mathrm{~cm})$ apresentaram melhores resultados no teste Arremesso da Medicine ball do que as crianças de $7(146,6 \mathrm{~cm})$ e 8 anos $(166,5 \mathrm{~cm})$.

Resistência Muscular Localizada. O fator Idade e a interação entre os fatores Grupo e Idade alcançaram níveis de significância, $F(3,54)=2,9930, p=0,03$ e $F(3,54)=7,0009$, $p<0,001$, respectivamente. 0 fator Grupo alcançou nível marginalmente significativo, $F(1$, 18) $=3,5665, p=0,07$. O Grupo Praticantes apresentou desempenho marginalmente superior no teste Abdominal em 1 minuto do que o Grupo Não-Praticantes. Independentemente do grupo, as crianças de 10 anos apresentaram resultado superior (20,7rep.) comparadas às crianças de 7 anos (14,2rep.).

Força de Membros Inferiores. Os fatores Grupo e Idade alcançaram níveis de significância, $F(1,18)=5,1311, p=0,03$ e $F(3,54)=11,995, p<0,0001$, respectivamente. 0 Grupo Praticantes apresentou desempenho superior no teste Salto Horizontal comparado

\begin{tabular}{l|l|l|l|}
\hline Ferracioli \& Nunes & 2018 & VOL. 12 & N.1 \\
\hline
\end{tabular}

6 of 12 
Brazilian Journal of Motor Behavior

ao Grupo Não-Praticantes. As crianças de $9(135,2 \mathrm{~cm})$ e 10 anos $(130,3 \mathrm{~cm})$ apresentaram desempenho superior ao das crianças de $7(104,5 \mathrm{~cm})$ e 8 anos $(113,1 \mathrm{~cm})$.

Resistência Aeróbia. Apenas o fator Idade alcançou nível de significância, $F(3,54)=4,0970, p=0,01$. As crianças de 10 anos apresentaram resultado superior no teste Corrida em 9 minutos $(1247,1 \mathrm{~m})$ comparadas com as crianças de $7(1090,7 \mathrm{~m})$ e 8 anos $(1080,8 \mathrm{~m})$.

A Tabela 4 apresenta a frequência relativa de crianças de cada idade e grupo que teve desempenho inferior ao típico, quanto ao desenvolvimento da coordenação motora, e inferior ao saudável, quanto ao desenvolvimento da aptidão física. Cinquenta porcento das crianças apresentaram desempenho abaixo do saudável no componente Força de Membros Superiores, 72,5\% no componente Resistência Muscular Localizada, 35\% no componente Força de Membros Inferiores e 36,2\% no componente Resistência Aeróbia. Apenas $17,5 \%$ das crianças apresentaram desenvolvimento da coordenação motora considerado em Risco ou com Dificuldade Motora.

Tabela 4. Frequência relativa (\%) de crianças participantes do presente estudo com níveis abaixo do Típico na avaliação da coordenação motora e abaixo do Saudável nos componentes da aptidão física avaliados.

\begin{tabular}{|c|c|c|c|c|c|c|c|}
\hline & Grupo & MABC & Flexibilidade & $\begin{array}{c}\text { Força Memb. } \\
\text { Superiores }\end{array}$ & $\begin{array}{c}\text { Res. Muscul. } \\
\text { Localizada }\end{array}$ & $\begin{array}{c}\text { Força Memb. } \\
\text { Inferiores }\end{array}$ & $\begin{array}{c}\text { Resistência } \\
\text { Aeróbia }\end{array}$ \\
\hline \multirow{2}{*}{7 anos } & Praticantes & 40 & - & 80 & 90 & 30 & 40 \\
\hline & Não-Pratic. & 10 & - & 40 & 60 & 20 & 30 \\
\hline \multirow{2}{*}{8 anos } & Praticantes & 40 & - & 40 & 50 & 40 & 50 \\
\hline & Não-Pratic. & 20 & - & 70 & 90 & 60 & 50 \\
\hline \multirow{2}{*}{9 anos } & Praticantes & - & - & 30 & 50 & - & 10 \\
\hline & Não-Pratic. & 20 & - & 30 & 90 & 30 & 50 \\
\hline \multirow{2}{*}{10 anos } & Praticantes & - & - & 50 & 60 & 40 & 20 \\
\hline & Não-Pratic. & 10 & 10 & 60 & 90 & 60 & 40 \\
\hline
\end{tabular}

\section{DISCUSSÃO}

O presente estudo analisou a influência da prática esportiva e da idade no desenvolvimento da aptidão física e coordenação motora de escolares de Fortaleza/CE. De maneira geral, os resultados mostraram que a idade, enquanto processos desenvolvimentais, influenciou o desempenho das crianças nas capacidades Destreza Manual, Equilíbrio, Força de Membros Superiores, Resistência Muscular Localizada, Força de Membros Inferiores, Resistência Aeróbia e na coordenação motora geral, enquanto a

\begin{tabular}{l|l|l|l|}
\hline Ferracioli \& Nunes & 2018 & VOL. 12 & N.1 \\
\hline
\end{tabular}


Brazilian Journal of Motor Behavior

prática esportiva influenciou o desempenho nas capacidades Equilibrio e Força de Membros Inferiores apenas.

Com relação ao desenvolvimento da Destreza Manual, a literatura aponta que a mão tem função de criar e interagir com o ambiente, caracterizando-se como uma extensão do intelecto. Além disso, seu desenvolvimento está diretamente relacionado com 0 funcionamento do córtex cerebral ${ }^{16}$. Desta forma, este componente motor representa um acúmulo de experiências no âmbito das ações motoras ${ }^{17} \mathrm{e}$, por isso, frequentemente pode aumentar com a idade. No entanto, as crianças de 8 anos apresentaram desempenho inferior às demais neste componente. Analisando individualmente, verificou-se $15 \%$ das crianças de 8 anos, em contrapartida a $5 \%$ das demais faixas etárias, com escore abaixo do $5^{\circ}$ percentil na Destreza Manual. A prática de atividades esportivas extracurriculares não influenciou o desempenho das crianças no presente estudo. Este fato deve estar relacionado ao tipo de modalidades praticadas pelas crianças (Tabela 1), que, de maneira geral, requerem habilidades motoras grossas. Ainda, a prática de atividades da vida diária e acadêmicas favorece 0 desenvolvimento da destreza manual ${ }^{17}$. Era esperado que a prática de atividades do cotidiano fosse realizada por todas as crianças participantes deste estudo, já que são de uma mesma cultura e, talvez por isso, as crianças não praticantes de atividades esportivas tenham apresentado desempenho marginalmente superior ao das crianças praticantes neste componente.

Também era esperado que as crianças praticantes de atividades esportivas extracurriculares apresentassem desempenho superior ao das não praticantes no componente Arremessar e Receber, pois, frequentemente, o desenvolvimento motor pode ser influenciado pela cultura esportiva e social da criança ${ }^{18}$. Pellegrini et al. ${ }^{18}$ afirmam que o desempenho em tarefas de arremessar e receber a bola é influenciado pela cultura de "bola" que a criança brasileira está inserida. No entanto, apenas $25 \%$ das modalidades praticadas (futebol, futsal, basquetebol e voleibol) requerem esse componente motor e, talvez por isso, não tenha sido observada diferença no desempenho das crianças participantes. Desta forma, sugere-se que as crianças do presente estudo podem estar vivenciando habilidades de arremessar e receber, tanto nas atividades curriculares como nas extracurriculares, de maneira semelhante.

O componente Equilíbrio é requerido no desempenho de diversas habilidades esportivas ${ }^{5}$, como aquelas que demandam mudanças de direção, manutenção da postura e alterações de velocidade. As atividades praticadas pelas crianças deste estudo demandam esse controle postural (Natação, Lutas, Ballet, Futebol/Futsal, Voleibol e Basquetebol) e, por isso, o Grupo Praticantes pode ter apresentado desempenho superior ao do Grupo Não-Praticantes. Estudos mostraram que crianças melhoraram seu equilibrio após um período de prática sistematizada de ballet clássico e futsal ${ }^{5}$ e que crianças praticantes de atividades radicais extracurriculares apresentaram melhor desempenho no equilíbrio dinâmico do que as não praticantes ${ }^{19}$. Também, as crianças não praticantes dessas modalidades foram consideradas com desempenho típico e, por isso, a influência dos processos desenvolvimentais estava interligada à experiência prévia das crianças ${ }^{19}$. Este aspecto pode ser observado no presente estudo, tendo em vista que as crianças mais velhas apresentaram desempenho superior ao das crianças mais novas no Equilibrio. Assim, fica evidenciado que tanto a prática esportiva extracurricular de modalidades que

\begin{tabular}{l|l|l|l|}
\hline Ferracioli \& Nunes & 2018 & VOL. 12 & N.1 \\
\hline
\end{tabular}


Brazilian Journal of Motor Behavior

requerem equilíbrio como o processo desenvolvimental das crianças participantes levaram ao desenvolvimento deste componente.

Os resultados da avaliação geral direcionam à compreensão de que a coordenação motora é desenvolvida ao longo dos anos e aprimorada em função deles ${ }^{20}$. Entende-se por processos desenvolvimentais as mudanças que os indivíduos passam ao longo da vida, que cada ser humano tem seu tempo próprio para aquisição das capacidades (de acordo com a maturação) e habilidades motoras (de acordo com as experiências) ${ }^{1}$. Desta forma, considera-se que todas experiências motoras vivenciadas pelas crianças favorecem 0 desenvolvimento motor. Não ficou evidenciado se experiências provenientes da prática esportiva extracurricular garantem desenvolvimento da coordenação motora superior àquele proveniente das atividades curriculares e da vida diária, pois a prevalência de crianças identificadas com Risco ou com Dificuldade Motora foi baixa e semelhante entre os grupos.

Com exceção do componente Flexibilidade, as crianças mais velhas apresentaram resultados superiores aos das crianças mais novas na avaliação da aptidão física. As características biológicas têm grande influência nos níveis de aptidão física de crianças e adolescentes, pois as variáveis neuromusculares (força/resistência) sofrem influência das transformações fisiológicas e anatômicas decorrente, também, das descargas hormonais, que são elevadas com 0 aumento da idade 6,21,22. Além disso, 0 crescimento somático e 0 aumento da massa muscular, em função do aumento da idade, fazem com que órgãos, como o coração e os pulmões, aumentem de tamanho, melhorando a captação e utilização do oxigênio'.

As crianças mais velhas apresentaram desempenho superior ao das crianças mais novas e as crianças do Grupo Praticantes apresentaram desempenho superior ao das crianças do Grupo Não-Praticantes no componente Força de Membros Inferiores. Ulbrich et al. ${ }^{21}$ mostraram aumento significativo no desempenho do teste Salto Horizontal em função do aumento da idade, devido a experiência com o movimento, ao tamanho corporal e ao comprimento das pernas dos indivíduos mais velhos. Além disso, as modalidades esportivas extracurriculares praticadas pelas crianças (Tabela 1) requerem força e agilidade ao executar movimentos com as pernas. Desta forma, a prática esportiva parece direcionar o desenvolvimento da aptidão física de acordo com as exigências da modalidade praticada 3 .

As crianças de ambos os grupos e de todas as idades apresentaram desempenho semelhante no componente Flexibilidade, corroborando com outros estudos ${ }^{21,23}$. Segundo Hiraga et al. ${ }^{24}$, o teste Sentar e Alcançar exige pouco componente motor durante sua execução. Assim, talvez a influência da prática esportiva e dos processos desenvolvimentais no desenvolvimento motor não seja evidenciada neste teste por ele não exigir padrões motores específicos.

Muitas crianças de todas as idades e grupos apresentaram desempenho abaixo do esperado nos componentes da aptidão física. Esse resultado sugere que, mesmo com melhora da aptidão física em função da idade, as crianças não estão alcançando seus níveis saudáveis. Ao contrário do que foi mostrado quanto ao desenvolvimento da coordenação motora, considera-se que as experiências das atividades curriculares e de vida diária não têm corroborado com o desenvolvimento da aptidão física das crianças e, assim, sugere-se que a prática de atividades físicas, recreativas e acadêmicas tenha atenção específica (de

\begin{tabular}{l|l|l|l|}
\hline Ferracioli \& Nunes & 2018 & VOL. 12 & N.1 \\
\hline
\end{tabular}


Brazilian Journal of Motor Behavior

pais e professores) e que garanta mudanças significativas nos níveis de desenvolvimento da criança.

Sugere-se que a prática esportiva pode influenciar capacidades físicas e motoras vivenciadas na modalidade praticada. No entanto, para esta constatação, é preciso investigar de maneira abrangente as características dos esportes. Os dados encontrados reforçam a literatura da área, embora direcionem a compreensão de que processos desenvolvimentais possuem maior influência no desenvolvimento motor do que atividades esportivas extracurriculares. Assim, professores de Educação Física e de Esportes que trabalham com crianças devem ter um conhecimento aprofundado sobre questões acerca da coordenação motora e aptidão física e suas relações com 0 processo de desenvolvimento motor.

Como limitação do estudo, é possível que a influência da prática esportiva sobre 0 desenvolvimento motor não tenha sido observada devido ao tamanho da amostra. Houve dificuldade para encontrarmos crianças, de 7-10 anos, assíduas e frequentes às práticas esportivas por quatro meses ou mais. Além disso, não se obteve informações sobre 0 volume da prática das modalidades (como dias praticados por semana e duração da prática). Acredita-se que as exigências do contexto (como tipo de modalidade praticada), as características da criança (inatas e adquiridas) e a quantidade de experiência influenciam 0 desenvolvimento motor. Desta forma, sugere-se que estudos futuros devem considerar estas variáveis no desempenho em testes de coordenação motora e aptidão física. Sugerese, ainda, que utilize-se uma bateria de avaliação mais indicada para coordenação motora grossa, e não MABC-2, na análise da influência da prática esportiva no desenvolvimento motor.

\section{REFERÊNCIAS}

1. Gallahue D, Ozmun JC, Goodway JD. Compreendendo o desenvolvimento motor: bebês, crianças, adolescentes e adultos. Porto Alegre: AMGH; 2013.

2. Newell KM. Constraints on the development of coordination. In: Wade MG, Whiting HTA, editors. Motor development in children: aspects of coordination and control. Dordrecht: Martinus Nijhoff; 1986. 341-60.

3. Nazario PF, Vieira JLL. Sport context and the motor development of children. Rev Bras Cineantropom Desempenho Hum 2014;16:86-95.

4. da Rocha PGM, da Rocha DJO, Bertolasce AL. A influência da iniciação ao treinamento esportivo sobre o desenvolvimento motor na infância: um estudo de caso. J Phys Educ 2010;21:469-77.

5. Santos CR, Silva CC, Damasceno ML, Medina-Papst J, Marques, I. Efeito da atividade esportiva sistematizada sobre o desenvolvimento motor de crianças de sete a 10 anos. Rev Bras Educ Fís Esporte 2015;29:497-506. 
Brazilian Journal of Motor Behavior

6. Bergman GG, Araújo MLB, Garlipp DC, Lorenzi TDC, Gaya A. Alteração anual no crescimento e na aptidão relacionada à saúde de escolares. Rev Bras Cineantropom Desempenho Hum 2005;7:55-61.

7. Guedes DP. Implicações associadas ao acompanhamento do desenvolvimento motor de crianças e adolescentes. Rev Bras Educ Fís Esporte 2007;21:37-60.

8. Sanches SM, Rubio K. A prática esportiva como ferramenta educacional: trabalhando valores e a resiliência. Educ Pesqui 2011;37:825-41.

9. Sa VW, Pereira JS. Influência de um programa de treinamento físico específico no equilíbrio e coordenação motora em crianças iniciantes no judô. Rev Bras Ciênc Mov 2003;11:45-52.

10. Machado Filho R, Pellegrinotti IL, Gonelli PRG. Crescimento e desenvolvimento das capacidades motoras de meninos escolares praticantes de atividade física geral. Acta Bras Mov Hum 2017;2:45-9.

11. Bortoni WL, Bojikian LP. Crescimento e aptidão física em escolares do sexo masculino, participantes de programa de iniciação esportiva. Braz J Biomotricity 2007;1:114-22.

12. Damico DM, Junqueira APR, Machado Filho R. Diferenças de velocidade e agilidade entre praticantes e não praticantes de futsal na fase pré-púbere da cidade de Petrópolis-RJ. Rev Bras Futsal Futebol 2013;5:99-104.

13. Henderson SE, Sugden DA, Barnett AL. Movement assessment battery for children-2. London: Harcourt Assessment; 2007.

14. Cenesp-Ufrgs. Projeto Esporte Brasil: Indicadores de saúde e fatores de prestação esportiva em crianças e jovens. [online] 2005; Disponível em: http://seer.ufrgs.br/index.php/perfil/article/download/77695/44409. [2016 jan 25].

15. Gaya A, Lemos A, Gaya A, Teixeira D, Pinheiro E, Moreira R. Projeto Esporte Brasil: manual de testes e avaliação. Porto Alegre: UFRGS; 2015.

16. Latash ML, Turvey MT. Dexterity and its development. New Jersey: Lawrence Erlbaum Associates; 1996.

17. Carmeli E, Patish H, Coleman R. The aging hand. J Gerontol 2003;58A:146-52.

18. Pellegrini AM, Souza Neto S, Hiraga C, Bellan P, Oliveira RB, Garcia Filho SM. Dificuldades motoras em crianças de 9-10 anos de idade: seriam os meninos mais descoordenados? In: Pinho SZ, Saglietti JR, editores. Núcleos de Ensino da UNESP. São Paulo: Cultura Acadêmica; 2008. 77-88.

19. Araújo MP, Barela JA, Celestino ML, Barela AMF. Contribuição de diferentes conteúdos das aulas de educação física no ensino fundamental I para o desenvolvimento das habilidades motoras fundamentais. Rev Bras Med Esporte 2012;18:153-7.

20. Collet C, Folle A, Pellozin F, Botti M, Nascimento JV. Nível de coordenação motora de escolares da rede estadual da cidade de Florianópolis. Motriz 2008;14:373-80.

21. Ulbrich AZ, Bozza R, Machado HS et al. Aptidão física em crianças e adolescentes de diferentes estágios maturacionais. Fit Perform J 2007;6:277-82. 
Brazilian Journal of Motor Behavior

22. Dumith SC, Ramires VV, Souza MJA et al. Aptidão física relacionada ao desempenho motor em escolares de sete a 15 anos. Rev Bras Educ Fís Esportes 2010;24:5-14.

23. Farias EDS, Carvalho WRGD, Gonçalves EM, Guerra-Júnior G. Efeito da atividade física programada sobre a aptidão física em escolares adolescentes. Rev Bras Cineantropom Desempenho Hum 2010;12:98-105.

24. Hiraga CY, Rocha PRH, Ferracioli MDC, Gama DT, Pellegrini AM. Physical fitness in children with probable developmental coordination disorder and normal body mass index. Rev Bras Cineantropom Desempenho Hum 2014;16:182-90.

Citation: Ferracioli, MC \& Nunes, LS. Influência da prática esportiva e da idade no desenvolvimento da coordenação motora e da adaptidão física de escolares de Fortaleza/CE. BJMB. 2018: 12(1): 1-12.

Editor: Joao A. C. Barros, California State University Fullerton, Fullerton, CA, USA.

Copyright: () 2015 Ferracioli, MC, Nunes and BJMB. Este é um artigo de acesso aberto distribuído sob os termos da Licença Internacional (Creative Commons Attribution-NonCommercial-NoDerivatives 4.0 International License) que permite uso, distribuição e reprodução irrestritos em qualquer meio, desde que 0 autor e a fonte originais sejam creditados..

Financiamento: Não houve financiamento para esse estudo.

Conflito de interesse: Os autores declararam não existir conflito de interesse.

Download: http://socibracom.com/bjmb/index.php/bjmb/issue/view/44 